\title{
MODELOS ALTERNATIVOS DE INFLACIÓN: ECONOMÍAS DE MERCADO Y ECONOMÍAS PLANIFICADAS*
}

\author{
JAN WINIECKI
}

Polish Institute of International Affairs

INTRODUCCIÓN

QUizÁ UN PUNTO DE PARTIDA tan válido como cualquier otro sea señalar lo que este ensayo no pretende ser. Mi propósito no es hacer una revisión de teorías de inflación ya conocidas. Dada la abundancia de literatura sobre economías de mercado y, por el contrario, la falta de literatura sobre economías planificadas, un ensayo con ese objetivo tendería a concentrarse demasiado en las primeras.

Simplemente, me limitaré a dar mi propia explicación sobre las fuentes de inflación y los procesos inflacionarios. Empezaré por las economías de mercado, estableciendo un marco analítico y jerarquizando las fuentes de acuerdo con su importancia a largo plazo. En segundo término, examinaré las economías planificadas siguiendo la misma pauta. En ambos casos destacaré una fuente de inflación como la más característica y persistente durante el periodo de posguerra.

\footnotetext{
* Traducción del inglés de Carlos Roces.
} 


\section{FUENTES DE INFLACIÓN EN ECONOMÍAS DE MERCADO:} OBSERVACIONES PRELIMINARES

Antes de analizar cualquier modelo específico, trataré de establecer claramente mi punto de vista sobre la inflación en economías de mercado. No comparto la idea de que la inflación tenga un origen único (como piensan, por ejemplo, los monetaristas). Las fuentes son de diversa indole. La inflación típica por presión de demanda, manifiesta, en general, bien avanzado el ciclo econúmico, es la más conocida y mejor estudiada. Por ocurrir periódicamente, se distingue de aquellos procesos inflacionarios que, causados por malas cosechas, guerras y otros fenómenos naturales o provocados por el hombre, se presentan de manera irregular. Ambos tipos de inflación, sin embargo, tienen un elemento importante en común. Pueden prolongarse más o menos tiempo, según su impacto, el patrón nacional de determinación de precios y salarios, el sistema impositivo, el mecanismo de indización, la cohesión social, etc., pero terminan por perder fuerza hasta borrarse de la escena económica. ${ }^{1}$

Por el contrario, hay otro tipo de inflación que, impulsada por la influencia permanente que ejercen en los precios complejos fenómenos tecnológicos, económicos y sociales relacionados entre sí, persiste a todo lo largo del ciclo económico.

Es este tipo de inflación el que mejor define el proceso inflacionario real del mundo occidental a partir de la II Guerra. Los rasgos más importantes de este proceso, desconocido hasta entonces por las economías de mercado, son los siguientes:

1) Su persistencia. El crecimiento anual de precios fue lento (al menos hasta 1972), pero incesante.

2) Su expansión transnacional. El incremento de precios se extendió a todas las economías de mercado industrializadas.

3) Tasas de inflación que aumentaron ininterrumpidamente. En general, las tasas anuales subieron de un quinquenio a otro o de un ciclo a otro.

Este tipo de inflación comenzó a manifestarse con claridad a mediados de la década del cincuenta, una vez que las economías

1 En mis trabajos anteriores no hacía distinción entre inflación por exceso de demanda e inflación por fenómenos que ocurren irregularmente. En este trabajo establezco la separación, ya que los modelos para explicar cada uno de dichos fenómenos difieren $\mathrm{y}$ son precisamente estos modelos el objeto del trabajo. 
industrializadas de Occidente habían superado las presiones inflacionarias de la guerra y la posguerra. El modelo seleccionado para analizarlo constituye la parte central del análisis hecho en este trabajo sobre las economías de mercado.

\section{EL ENTORNO ECONÓMICO Y SOCIAL DE LA POSGUERRA: CONCATENACION ENTRE PRODUCTIVIDAD, GANANCIAS, COSTOS, SALARIOS Y PRECIOS ${ }^{2}$}

El modelo que, en mi opinión, explica la persistencia, la transnacionalización y el aumento ininterrumpido de las tasas de inflación en economías de mercado industrializadas puede incluir dos grandes sectores: por ejemplo, la industria (minería, manufacturas, construcción, energía) y los servicios. El sector agrícola queda fuera por tener poco peso en el producto nacional de las economías industrializadas de Occidente y, sobre todo, porque sólo ejerce presión inflacionaria a través de fenómenos esporádicos (malas cosechas, por ejemplo). ${ }^{3}$

Destaquemos, primero, un rasgo común a las economías industrializadas, aunque extensivo, como se sabe, a las economías en desarrollo. Se trata de las diferencias que observa la tasa de crecimiento de la productividad tomando los distintos sectores y ramas de la economía. Esta heterogenidad ha sido a menudo pasada por alto al explicar las causas de la inflación (algunas excepciones son: $P$. Streeten, 1962, W. J. Baumol, 1967, M. Kucharski, 1969, los autores que diseñaron el modelo conocido como EFO, 1969, O. Aukrust, 1970 y J. A. Pewzner, 1975 y 1976).

Por razones examinadas más adelante, las diferencias en crecimiento de productividad más importantes se dan en la industria y, particularmente, en las manufacturas. El desarrollo histórico de las economías capitalistas de mercado revela, sin embargo, que estas diferencias no dan origen a presiones inflacionarias hasta que se producen los grandes cambios del siglo $\mathrm{XX}$.

2 El modelo descrito brevemente en esta sección ha sido presentado en tres trabajos míos publicados en inglés, polaco y francés, véase J. Winiecki, $1978,1980,1982$.

3 Naturalmente, la política agrícola de determinados países o grupos de países, por ejemplo, la CEE, matiza el impulso inflacionario que significan los precios agrícolas, pero, incluso de esta manera, esta presión es de carácter distinto que las analizadas en el modelo. 
Gran proporción de las ramas industriales que registran tasas de crecimiento de productividad altas operan bajo una estructura de mercado oligopólica que influye notoriamente en la determinación. de precios y salarios. Puesto que en oligopolio (o monopolio puro) la competencia de precios no es un recurso habitual, la tasa de crecimiento de productividad en esas ramas no lleva a una disminución de precios. El crecimiento de productividad por encima del promedio se traduce en un aumento de ganancias.

Al aumentar las ganancias se fortalece el poder de negociación salarial que tienen los sindicatos. Dado el nivel de precios, un alza de salarios es fácilmente conciliable con ganancias moderadamente más altas. ${ }^{4}$ Por consiguiente, en ramas donde la productividad es más dinámica, la tasa de crecimiento de salarios es igual o ligeramente menor que la tasa de crecimiento de la productividad. ${ }^{5}$ Esta relación se da bajo condiciones normales, o sea, cuando no hay presiones inflacionarias fuertes de otro tipo que muevan a los salarios a crecer a la par que los precios.

El hecho de que los salarios no aumenten a una tasa mayor que la productividad precisamente en aquellas ramas donde la tasa de crecimiento de la productividad es alta lleva a poner en duda la hipótesis, sostenida por algunas teorías de inflación, de que los salarios, como norma, aumentan más rápidamente que la productividad. El crecimiento de salarios en las ramas con productividad más dinámica no representa en sí mismo una presión inflacionaria.

Sin embargo, en la posguerra las relaciones económicas y sociales del mundo occidental dieron un giro importante. Cambió el patrón que guía cómo remunerar a la mano de obra imponiéndose el principio de que los salarios de todos los sectores de la economía aumentaran en un mismo porcentaje, sin tomar en cuenta diferencias de productividad. ${ }^{6}$ Esta norma de equidad horizontal fue aceptada socialmente a partir del propósito mismo que la anima.

No es difícil imaginar la presión inflacionaria que supone una tasa de aumento de salarios igual para todos los sectores y ramas industriales cuando la productividad crece de manera desigual. En

4 Véase M. Kalecki, 1956.

5 Para pruebas empíricas sobre esta conclusión, véase J. Eatwell, J. Lewellyn y R. Tarling, 1974 y J. Winiecki, 1980.

6 Hay otros aspectos del patrón de remuneración de la mano de obra -diferenciales por habilidades específicas, grado de calificación, antigüedad, responsabilidad e intensidad del trabajo- que no tomaremos en cuenta en este trabajo, aunque, como se apuntará, también inciden en la inflación. 
cada una de las ramas el costo unitario de la mano de obra aumentará a una tasa igual a la diferencia entre el incremento general en salarios y el aumento particular en productividad. ${ }^{7}$ En los servicios el efecto será todavía mayor, puesto que la productividad crece más lentamente que en la industria (en algunos subsectores, como la administración pública, el crecimiento es cero, por definición).

Tarde o temprano el aumento de costos se traduce en aumento de precios. Es así como el alza de salarios provoca aumento de precios, no en las ramas de mayor dinamismo en productividad, donde justamente esta ventaja actúa como freno, sino en el resto de la industria y en los servicios.

Al llegar a este punto surgen dos interrogantes. Habría que preguntarse, en primer término, por qué los trabajadores o los organismos que los representan rechazan todo aumento de salarios proporcionalmente menor que el de la productividad. La respuesta está en la presencia de oligopolios. Esta forma de competencia, como señalabamos, impide que los aumentos en productividad se traduzcan en disminuciones de precios. De bajar los precios, por lo demás, el beneficio sería para la sociedad en general y no para los trabajadores en particular. Los trabajadores sólo pueden beneficiarse directamente de aumentos en productividad por encima del promedio logrando que los salarios aumenten allí donde prestan sus servicios.

En segundo término, cabría examinar si los precios suben, efectivamente, sólo en aquellas ramas cuya naturaleza oligopólica les permite pasar el aumento de costos a los consumidores, o bien, incluso, en las ramas bajo competencia más abierta. Como es bien sabido, un aumento de costos generalizado (que no afecta únicamente al productor individual de una rama industrial) se carga rápidamente a los precios. Cada productor eleva su precio sabiendo que sus competidores, por verse afectados como él, no podrán actuar de modo distinto a largo plazo. El momento que las ramas más competitivas escogen para subir precios depende, sin embargo, de la posición cíclica de la economía. Las ramas competitivas y las ramas oligopólicas no elevan precios simultáneamente.

El encadenamiento entre productividad, ganancias, salarios, costos y precios genera procesos inflacionarios que pueden alargarse indefinidamente. Al subir los salarios de manera uniforme, el aumento consiguiente de precios en casi todos los servicios y en aquellas ramas

7 No se considera la posibilidad de un alza proporcional en el margen de ganancia porque no está contemplada en ningún modelo. 
industriales cuya productividad crece a una tasa igual o menor que la tasa promedio de la industria, borra en parte el aumento en el poder de compra del salario que hayan logrado los trabajadores de las ramas con productividad más dinámica al imponer originalmente un alza de su salario. Ello puede ser motivo de un nuevo incremento uniforme de salarios, pero la causa decisiva del primero es el aumento de ganancias en las ramas industriales donde la productividad crece a tasa mayor.

En condiciones normales, las ramas con productividad más dinámica permiten que sus salarios aumenten a la misma tasa o a una tasa ligeramente menor que su productividad y, si operan bajo competencia oligopólica, rara vez pasan a los precios este incremento de salarios. El comportamiento de estas ramas provoca que los trabajadores del resto de la industria y de los servicios demanden mayor remuneración. El incremento general de salarios termina por ser igual al otorgado por las ramas más dinámicas y las diferencias en crecimiento de productividad se traducen en mayores costos laborales unitarios y, después de cierto tiempo, en mayores precios.

Este modelo explica sucintamente la persistencia de la inflación en las economías de mercado contemporáneas y su extensión a todos los países industrializados de Occidente, que, por similitud de condiciones sociales, tienen el mismo patrón de determinación de salarios. ${ }^{8}$

Hasta aquí nos hemos referido al origen de la inflación. Hemos dejado de lado la tasa a que crecen los precios. Sin embargo, nuestro esquema nos permite definir las determinantes de esta variable:

a) Las diferencias en crecimiento de productividad, tomando, por una parte, las ramas de productividad más dinámica (que fijan el aumento general de salarios) y, por otra, el conjunto de la industria y los servicios.

b) El peso específico que tienen en el PIB la industria y los servicios.

c) La combinación de $a$ ) y $b$ ).

Claramente, conforme mayores son las diferencias en crecimiento de productividad y mayor el peso de los servicios en el PIB, más alta es la tasa de inflación de un país. ${ }^{9}$ Mediante este razonamiento po-

8 Para evidencia empírica, véase J. Eatwell, J. Llewellyn y R. Tarling, 1974 y J. Winiecki, 1980.

9 Para un modelo formal que calcula el aumento de costos debido a las causas señaladas en el texto, véase J. Winiecki, 1980. 
demos explicar, también, la tercera caracteristica de la inflación de posguerra, o sea, el aumento incesante de la tasa de crecimiento de precios. Este fenómeno se debe a que las diferencias en crecimiento de productividad se han profundizado o a que el sector de productividad menos dinámica (servicios) cobra cada vez más importancia. Aunque falta evidencia empirica que apoye estas conclusiones, es claro que, en las economías de mercado, el peso del sector servicios en el PIB aumenta lentamente pero a velocidad uniforme. Además, según diversos cálculos, entre 1958-1971 y 1971-1975 se observa un aumento promedio de las diferencias en crecimiento de productividad, tomando, por una parte, como ya señalábamos en párrafos anteriores, las ramas industriales más dinámicas en este aspecto $y$, por otra, el conjunto de la industria y los servicios. ${ }^{10}$ Este hecho puede tomarse como presión adicional sobre la tasa de inflación.

Las relaciones causales que se han desprendido de dicha información pueden tomarse como mero empirismo, pero hay otros elementos del modelo que se han probado formalmente. De una parte, mientras que las tasas de crecimiento del salario son similares, las tasas de crecimiento de la productividad muestran ser desiguales. Registran coeficientes de dispersión (desviación estándar y coeficientes de variabilidad) mucho mayores. De otra parte, tomando los años 1958-1967 y 1958-1971 y un grupo de 14-15 economías de mercado en el primer período y de 12-14 en el segundo, las tasas de crecimiento de productividad explican el $60 \%-80 \%$ y el $55 \%-80 \%$, respectivamente, de las diferencias en crecimiento de salarios entre una economía y otra. Los resultados son significativos a niveles de 0.01 y de 0.001. Sin embargo, en el periodo 1971-1975, cuando la reivindicación salarial para recuperar poder de compra empieza a tener importancia, las tasas de crecimiento de productividad sólo explican el $20 \%-30 \%$ de las diferencias en crecimiento de salarios, aunque, en este caso, los resultados sólo sean parcialmente significativos y a niveles de 0.05 y $0.10 .^{11}$

Cabe señalar, también, que para los períodos 1958-1971 y 19711975 las tasas de crecimiento de costos generadas por el modelo explicaron, respectivamente, el $87 \%$ y el $56 \%$ de las diferencias observadas en 14 economías de mercado (las razones $F$ fueron 14.155

10 J. Winiecki, 1980.

11 Véase J. Eatwell, J. Llewellyn y R. Tarling, 1974 y J. Winiecki, 1980. El segundo de los trabajos contiene las pruebas para 1971-1975. 
y 2.88). Estos resultados son significativos a nivel de 0.001 para el primero de los periodos únicamente. Las variables consideradas como independientes en el modelo, son: la diferencia en crecimiento de productividad, tomando, por una parte, las ramas industriales de productividad más dinámica $\mathrm{y}$, por otra, el resto de la industria; la diferencia en crecimiento de productividad, tomando, por una parte, el conjunto de la industria $y$, por otra, los servicios; el peso de la industria en PIB y el peso de los servicios en el PIB. Las estimaciones abarcaron al grupo de las 14 economías.

Hay dos tesis muy discutidas que debieran ser examinadas a la luz de las consideraciones hechas hasta aquí. La primera y sostenida hace más tiempo es una variante de la idea de Galbraith de que las empresas y las agrupaciones laborales más poderosas tienen la posibilidad de pasar a los consumidores todo aumento de costo de producción resultante del alza de salarios. En otras palabras, que las grandes corporaciones, por tener poder oligopólico en sus respectivas industrias, incrementan precios (e incluso, a veces, márgenes de ganancia) al verse afectadas por aumentos de salarios. De ahí que el remedio prescrito sea controlar la política de precios de las empresas grandes, cuya iniciativa, se piensa, mueve a las más pequeñas. ${ }^{12}$

Si evaluamos esta tesis a partir del análisis expuesto en páginas anteriores, concluiremos que su error es confundir el efecto del problema con la causa. Los acuerdos salariales que generalmente sirven de guía (por ejemplo, los de la industria del acero, del automóvil, del hule, etc.) no son el acicate de una espiral inflacionaria, sino el reflejo. La espiral brota de las ramas industriales con mayor crecimiento de productividad. Como las industrias en que se negocian los acuerdos salariales clave son oligopolios con baja tasa de crecimiento de productividad, al tener que otorgar el aumento de salarios que fijan las ramas con productividad más dinámica sufren un alza de costos que trasladan a precios. Si sólo se indica el efecto de que haya tasas de crecimiento de productividad distintas que deben coexistir con una tasa de crecimiento de salarios uniforme (fijada, además, por las ramas de productividad más dinámica), la causa del proceso inflacionario queda encubierta.

La segunda tesis, muy difundida durante los setenta y contenida, por ejemplo, en la llamada "curva de Laffer", asocia la infla-

12 Véase, J. K. Galbraith, 1976. Algunos autores de la llamada escuela estructuralista hacen un planteamiento en la misma línea. Consúltese, por ejemplo, G. C. Means, 1975 y J. M. Blair, 1975. 
ción y el bajo crecimiento económico a la expansión del sector público. Aquí, el error consiste en confundir una parte con el todo. De acuerdo con nuestro esquema, la tasa de inflación no sube porque crece el sector público en particular. Sube porque crece el sector servicios en conjunto y no hay compensación por el lado de una disminución de las diferencias en crecimiento de productividad. El sector público es parte del sector servicios y, por tanto, alcanza un crecimiento de productividad relativamente más bajo que el de la industria. Es el conjunto del sector servicios, no sólo una parte, el que debe asociarse a la inflación.

\section{OTROS MODELOS DE INFLACIÓN EN ECONOMÍAS DE MERCADO}

Señalábamos al comenzar el trabajo que la inflación tiene varios orígenes. Incluso suponiendo que el modelo esbozado en la sección anterior explique lo fundamental del proceso inflacionario real hasta principios de los setenta y algunos de sus elementos más importantes en el período a partir de entonces, es necesario tener en cuenta otros esquemas.

Generalmente, en períodos de auge, la inflación típica por presión de demanda se añade a la inflación persistente definida en nuestro modelo. Por ello, al menos hasta 1971, la inflación contemporánea muestra, como otro de sus rasgos característicos, mayor celeridad en períodos de auge que en periodos de recesión. A partir de 1972-1975 se rompe este patrón cíclico debido al fuerte impacto que causan fenómenos esporádicos ocurridos, en su mayoría, durante los años de recesión. Cabe pensar, sobre todo, en los aumentos de precios del petróleo.

Pero volvamos a modelos que se centran en la inflación persistente, aunque ninguno parezca tener la generalidad y el poder explicativo del que se ha presentado. El conflicto social se asocia a la inflación. Tanto la teoría marxista sobre este fenómeno como la llamada teoría sociológica francesa de inflación lo consideran como un elemento de presión permanente para cambiar el patrón de distribución del ingreso vía aumentos de salarios no justificados en términos de productividad. ${ }^{13}$

13 Dentro de la teoría marxista, uno de los trabajos que defienden esta postura es: W. Sadzikowski, 1977. Dentro de la escuela francesa, se pueden citar: H. Aujac, 1950 y J. Le Bourua, 1950. 
La teoría del conflicto social contribuye a explicar por qué se prolongan los procesos inflacionarios. La causa está en la reacción que provoca en determinados grupos o clases el intento de cambiar el patrôn de distribución del ingreso incrementando salarios. El esque$\mathrm{ma}$, sin embargo, es todavía demasiado general para explicar las causas mismas del proceso inflacionario en las economías de mercado. La sola existencia de conflicto social no basta para definir las características específicas de este proceso. El conflicto social es un fenómeno asociado de origen al sistema capitalista, aunque no siempre se haya manifestado con la misma intensidad, pero la inflación, por lo menos hasta mediados de los cincuenta, no tuvo nunca las características que ha mostrado en el presente.

No obstante, hay una causa de inflación persistente que sí queda apuntada. En algunas economías capitalistas, sobre todo en aquellas donde el ingreso está distribuido más inequitativamente, la agudización del conflicto social significa una presión constante sobre el salario. La inflación no depende del conflicto social en sí, sino del grado en que se manifiesta. Conforme más se agudiza el conflicto, mayor es la presión para aumentar salarios al margen de cambios en productividad y, por tanto, más alta es la inflación.

La duda es cómo medir la intensidad del conflicto social, aunque sólo sea a escala ordinal. Tratándose de un fenómeno tan complejo son necesarias ciertas simplificaciones. Algunas variables lo captan mejor que otras, sobre todo, si no se refieren a su manifestación (como es el caso con la huelga, por ejemplo), sino a sus causas, entre las cuales destaca, precisamente, la distribución del ingreso (la variable concreta a tomar aqui sería, digamos, el porcentaje que representa el quintil más bajo del ingreso nacional). Al observar la tasa de inflación de una economía de mercado, cualquier residuo por encima del promedio después de deducir la parte que explica el modelo descrito en este trabajo, pudiera adjudicarse a la presencia de conflicto social.

El aceleramiento de la inflación al finalizar los sesenta y durante la década siguiente tuvo una causa adicional. En algunas economías de mercado, el esfuerzo de sindicatos y algunos partidos por reducir diferenciales de salarios incrementando con particular ímpetu el salario mínimo y otros de bajo nivel ha topado con la resistencia activa de trabajadores calificados y empleados asalariados $\mathrm{y}$, por tanto, provocado una espiral salarios-salarios. Los países con sindicatos descentralizados tienen mayor posibilidad de enfrentar un proceso de esta índole. El Reino Unido es el mejor, o para el caso el peor, ejemplo. 
Cada una de las causas de inflación examinadas hasta ahora ha tenido distinto peso según el período que se tome. Desde mediados de los cincuenta hasta principios de los setenta, el aumento de precios sufrido por las economías de mercado debe asociarse a la concatenación entre productividad, ganancias, salarios, costos y precios. Dada la estabilidad característica de este período, sólo en algunos países hubo conflicto social que generara presión adicional. A fines de los sesenta la inflación tradicional estimulada por demanda empezó a tener mayor importancia que en períodos de auge anteriores y la espiral salarios-salarios se convirtió por primera vez en acicate inflacionario.

El panorama cambió a mediados de los setenta: Durante el ciclo económico 1972-1975, fenómenos aleatorios elevaron notoriamente la tasa de inflación, como ya se señaló, provocando, con ello, una aparente discontinuidad en un proceso limitado hasta entonces a un aumento lento en la tasa de inflación. Esos fenómenos se convirtieron en la causa principal del alza de precios y los aumentos compensatorios en salarios comenzaron a cobrar importancia.

\section{FUENTES DE INFLACIÓN EN ECONOMÍAS PLANIFICADAS: OBSERVACIONES PRELIMINARES}

A mediados de los sesenta algunas publicaciones especializadas de Europa Oriental comenzaron a difundir trabajos sobre inflación en economías planificadas, reflejando, con ello, la necesidad de fundamentar teóricamente un fenómeno ya entonces visible. ${ }^{14}$ Los trabajos publicados en ese período y algunos anteriores hechos por especialistas occidentales $^{15}$ giran casi exclusivamente en torno a la inflación tradicional por exceso de demanda. Este sesgo no sería criticable si no hubiera pasado por alto la causa más importante de este tipo de inflación cuando se produce en economías planificadas.

Una economía planificada se rige por mecanismos claramente distintos de aquellos que mueven a una economía de mercado. Por tanto, un fenómeno como la inflación por presión de demanda debe tener raíces diferentes en un ámbito y en otro. El exceso de demanda es, efectivamente, una causa de inflación en economías planificadas, 1969.

14 Cabe mencionar: J. Beksiak, 1966, J. Kornoi, 1971 y M. Kurcharski,

15 Véase, por ejemplo, P. J. D. Wiles, 1962. 
pero los esquemas que, en este contexto, sólo ponen de relieve el impacto expansionista que supone en la esfera de la producción el sistema de organización económica estudiado, pasan por alto un fenómeno importante, a saber, la fluctuación cíclica de la brecha inflacionaria (y de la inflación). Además de la presión que ejerce en la producción el sistema que ahora nos ocupa, hay otras fuerzas en juego.

En mi opinión, el elemento definitorio de la inflación por demanda en economías planificadas es el cambio cíclico (el aumento alternado por el descenso) en la escala de la brecha inflacionaria. Este fenómeno nace de un exceso de demanda en la esfera de la inversión y no en la esfera de la producción. Además, hay que tener en cuenta los múltiples encadenamientos entre brecha inflacionaria e inflación, entendida ésta como un aumento en el costo de la vida. En este trabajo expondré un modelo de inflación por exceso de demanda que destaca, por una parte, la concatenación entre instrumentos de planificación de inversión a mediano plazo, ciclos de inversión observada, desequilibrio global y brecha inflacionaria y, por otra, las formas en que la brecha inflacionaria se transforma en inflación, incluso bajo mecanismos tradicionales de control de precios.

El modelo servirá para examinar la mayoría de los distintos planteamientos sobre inflación en economías planificadas. Esta evaluación es importante, ya que, si bien, en mi opinión, el exceso de demanda es, efectivamente, la causa principal del problema, la manera como yo lo explico difiere de la interpretación tradicional. No dejaré de lado otras fuentes de inflación. Las describiré en menor o mayor detalle según hayan side tomadas en cuenta o no y, además, trataré de jerarquizarlas.

\section{LA EXPLICACIÓN TRADICIONAL DE LA INFLACIÓN POR EXCESO DE DEMANDA EN UNA ECONOMÍA PLANIFICADA}

Antes de evaluar el modelo señalado, examinemos la explicación tradicional sobre inflación por exceso de demanda en economías planificadas. La tesis central es que el carácter expansionista de los programas de organización económica provoca desequilibrio $\mathrm{y}$, por tanto, inflación. Así, según algunos libros de tex to, ${ }^{16}$ la economía planificada ha estado históricamente atrapada en sistemas de direc-

16 Por ejemplo, R. Bauer y otros, 1972. 
ción que inducen a la expansión continua de la producción y, por tanto, de la demanda de factores productivos y de insumos materiales, sin tomar en cuenta la relación costo-precio.

Este afán de expansión ha cobrado forma concreta tanto en los instrumentos de política administrativa (que tienen carácter impositivo), como en los instrumentos (o parámetros) de política económica. En su consolidación ha tenido un papel clave el hecho de que los incentivos a directores de producción o a trabajadores estén relacionados positivamente con el volumen o el valor de la producción, pero no con los costos de producción.

Al elaborar sus planes de producción, las empresas demandan factores productivos e insumos materiales casi sin límite, incluso cuando el costo de producción exceda el valor de la producción. Tal conducta está amparada en la convicción de que cualquier déficit de valor sobre costo lo cubrirán los superiores de las empresas dando subsidios o aumentando precios. Este exceso de demanda de factores e insumos, a partir de incentivos que empujan a las empresas a aumentar sus necesidades ilimitadamente ha sido llamado acertadamente "restricción holgada de presupuesto".17

Como toda empresa actúa de la misma manera, sabiendo, además, que la norma es general, surgen problemas adicionales. Para reducir al mínimo el riesgo de no entregar a tiempo el producto o de tener fallas de maquinaria - poniendo así en juego las metas de producción-, la empresa acumula excesivas cantidades de mano de obra, insumos y refacciones para su equipo. Este "instinto de ardilla" infla todavía más una demanda ya de por sí excesiva.

El modelo tradicional de inflación en economías planificadas no distingue entre sector de bienes de producción y sector de bienes de consumo, puesto que ambos compiten por la misma cantidad limitada de recursos. Pero esta restricción amplía todavía más la brecha inflacionaria. El sector de bienes de consumo, al no tener las facilidades de abastecimiento que tiene su competidor, prioritario en las decisiones de los planificadores, enfrenta mayores problemas para poner en práctica sus planes. ${ }^{18}$

Esta explicación acierta a ubicar el origen de la brecha inflacionaria persistente que sufren las economías planificadas. Dado el carácter permanente del sistema de dirección expansionista, la brecha persiste durante todo el periodo de planeación. El error, a mi juicio,

17 J. Kornoi, 1979.

18 J. Kornoi, 1979. 
es considerar que sea constante. El modelo tradicional trata al sector de inversión como si operara de la misma manera que el conjunto de la producción.

\section{EXCESO DE DEMANDA DE INVERSIÓN Y CICLOS DE INVERSIÓN ${ }^{19}$}

El exceso de demanda de inversión en economías planificadas ha sido un problema examinado exhaustivamente en la literatura económica desde principios de los sesenta. ${ }^{20}$

Según todas las interpretaciones, el fenómeno surge al iniciarse el periodo de industrialización acelerada, estrategia que, bajo la premisa de una relación lineal entre inversión y crecimiento económico, guiaba todo incentivo a incrementar la demanda de inversión. El mensaje era transmitido por canales formales o informales (por ejemplo, la opinión de cualquier superior) y llegaba a todas las instancias empresariales, desde las propias unidades de producción hasta los ministerios. Este estímulo a la producción sin tomar en cuenta los costos correspondientes derivó en un exceso de demanda de inversión persistente en cada uno de los periodos bajo plan económico.

La explicación omite un punto importante, relacionado con el origen mismo del fenómeno que trata. El sistema de organización de la producción pudo, efectivamente, llevar a las autoridades superiores a poner en primer plano un crecimiento a cualquier costo, pero ipor qué los dirigentes de las empresas, como norma, optaron por alcanzar esta meta limitándose a incrementar la inversión, particularmente la inversión en nuevo equipo? Como es bien sabido, los responsables de las empresas son muy reacios a implantar, por ejemplo, innovaciones técnicas o incluso de manejo empresarial. De esta manera, el objetivo de hacer crecer la producción se convirtió en el objetivo de hacer crecer la inversión.

Se señala a veces que la inversión es el recurso más fácil, es decir, el método de crecimiento menos costoso para la empresa que invierte. A pesar de que la inversión ha dejado de ser un recurso exento de costo en las economías planificadas, gracias, en parte, a ciertas reformas, continúa siendo el método de crecimiento más favorecido. Los

19 El modelo a que se dedica esta sección y parte de la siguiente aparece en Ekonomista, núm. 1, 1982.

20 Para una revisión en inglés de los primeros debates sobre este punto, véase A. Bajt, 1971. 
recortes a inversiones excesivas son un fenómeno tan común en el presente como en el pasado.

La persistencia en economias planificadas de una demanda de inversión excesiva, no obstante las múltiples reformas de los años sesenta y setenta, muestra que no basta con revisar los métodos de planeación y ejecución de la inversión. Hay que averiguar por qué determinado sistema de organización económica implica relaciones complejas que llevan a determinada actitud frente a la inversión.

En mi opinión, el exceso de demanda de inversión no se debe a un atractivo absoluto de dicho recurso, sino a un atractivo en términos relativos. Concretamente, un atractivo más fuerte que el de los métodos de crecimiento basados en innovaciones.

Cuando las empresas evalúan sus posibilidades de crecimiento, el objetivo es reducir al mínimo el riesgo de no cumplir las metas básicas formuladas en volumen o en valor del plan económico en curso y de los planes futuros. Conforme menor es este riesgo, menor es la probabilidad de no recibir los premios y bonos unidos a tales logros (y, también, de perder la estima de autoridades superiores). En este contexto, la forma de expansión segura es la inversión, sobre todo, la inversión en equipo nuevo. Toda innovación, técnica $u$ operacional, se incorpora a una planta productiva que está ya en funcionamiento. El personal que opera este equipo está interesado, más que nada, en cumplir las metas correspondientes al periodo que esté transcurriendo. El sistema de incentivos empuja en tal dirección. Incluso si la innovación asegura un incremento de producción, puede requerir un periodo de adaptación tan largo que afecte los programas de producción en curso. La sola posibilidad de ello lleva a los responsables de las unidades productivas a incrementar la producción presionando en la inversión. Además, una innovación que sí eleve la productividad en el período de producción en curso puede, como ya ha ocurrido, marcar metas más ambiciosas en el período siguiente y ser, por ello, contraproducente para los responsables de producción y los trabajadores, quienes llegado el momento, tendrían que poner mayor esfuerzo en alcanzar los premios y bonos asociados al cumplimiento del plan. La expansión de capacidad productiva por vía de equipo nuevo, en contraste con los demás métodos señalados, no supone gran esfuerzo y, sobre todo, ningún riesgo. La instalación de cualquier nueva planta o la ampliación de las ya existentes no afectan en nada la capacidad productiva instalada ni, por tanto, los programas de producción asociados a ella. Es decir, no ponen en peligro la ejecución del plan que esté en curso. 
El riesgo como elemento de análisis sirve, también, para entender por qué se prefiere equipo nuevo a equipo limitado a modernizar el ya existente, aunque sea menos costoso. Esta actitud se explica a veces como una atadura psicológica a la fase inicial de la industrialización en economías planificadas, cuando toda planta nueva se tomaba como símbolo de un país que avanzaba rápidamente en dicho proceso. La preferencia por equipo nuevo puede estar ligada a este fenómeno, pero tiene una explicación sólida en términos puramente económicos. Toda inversión complementaria, encaminada sólo a modernizar equipo ya instalado, como toda innovación no basada en inversión, afecta la capacidad en uso y, por consiguiente, supone el mismo tipo de riesgo que se señaló líneas atrás. De ahí que tenga menor atractivo que la inversión en equipo nuevo.

Hay un hecho adicional que propicia exceso de demanda de inversión. Para la empresa, la inversión representa un costo bajo, no en términos absolutos, sino en términos relativos. Esta propiedad no desaparecería aunque los proyectos que continúan estando exentos de costo (para la empresa, naturalmente) fueran eliminados. La doble paridad de precios establecida a comienzos de la industrialización impuso a los bienes de inversión una tasa de ganancia menor que a los bienes de consumo y provocó, así, una distorsión de precios que se ha mantenido a través del tiempo. La subvaluación de la maquinaría y el equipo deprime artificialmente los costos de inversión. La hace aparecer como una alternativa más redituable de lo que realmente es. A pesar de las críticas de que ha sido objeto durante por lo menos un cuarto de siglo, ${ }^{21}$ la doble paridad no parece estar llamada a desaparecer sino muy lentamente..$^{22}$

Hasta ahora hemos explicado por qué las empresas llamadas a expandir su producto optan por la inversión y no por la innovación. Examinemos, ahora, cómo esta tendencia se traduce en un patrón cíclico de expansión y contracción de la inversión.

No hay duda de que el ciclo es importante, pero no me parecen satisfactorios los argumentos más conocidos para explicarlo. No comparto, por ejemplo, la teoría de "ciclos de reinversión" formulada por Lange, donde el elemento principal es la falta de coincidencia en

21 Véase, por ejemplo, J. Malishev, 1957.

22 Cabe hacer notar que Hungría inició en 1980 un programa de reformas tendiente a eliminar hacia 1985 todo vestigio de doble paridad. Ya hace tiempo que este país se avoca seriamente a la tarea de cambiar la estructura de precios interna. 
el tiempo observada en los procesos de obsolescencia de las distintas partes de la capacidad instalada, ${ }^{23}$ o la teoría sobre la desproporción que guardan las tasas de crecimiento de la inversión y la producción registradas en las manufacturas frente a las que alcanzan las actividades extractivas. ${ }^{24}$ Es cierto que, a veces, los proyectos de reinversión no coinciden en el tiempo y que el ritmo a que invierten las actividade extractivas frena la expansión de las manufacturas, pero todos estos fenómenos son esporádicos y, por consiguiente, no explican el carácter recurrente de los ciclos de inversión. Este nace del expansionismo inherente al sistema de dirección que rige a las economías planificadas.

Comencemos por examinar el proceso que lleva a la elaboración de un plan de inversión a mediano plazo. A mi juicio, es en el diseño y en la ejecución de los planes de inversión, bajo el sistema de dirección señalado, donde hay que buscar la causa del patrón cíclico que nos proponemos explicar. La empresa presenta sus demandas de inversión bajo la forma que mejor asegure los fondos requeridos. De ahi que, a menudo, subestime los costos o sobreestime los resultados del proyecto que somete a consideración. Es el viejo recurso de utilizar el plan como "anzuelo", sabiendo de antemano que, llegado el momento, cualquier sobregiro en los costos presupuestados será cubierto con fondos adicionales, puesto que siempre se puede ejercer presión con el argumento de que es la economía nacional la que resiente las pérdidas de todo proyecto de inversión inconcluso. ${ }^{25}$ Esta manera de proceder es, por cierto, una manifestación más de lo que Kornoi llama "restricción laxa de presupuesto". Al iniciar su recorrido por las distintas instancias que, con diversa jerarquía, se inscriben en una economía planificada, las solicitudes de las empresas forman ya un paquete. Los límites que los planificadores centrales hayan marcado a los ministerios o los ministerios a las asociaciones habrán sido en buena medida pasados por alto, ya que cada organismo, movido por el sistema de incentivos, opera bajo la meta de expandir la producción. Generalmente, el paquete de solicitudes de inversión que reúne cada periodo la autoridad central excede en mucho los fondos (y la capacidad productiva) previstos para la inversión del período siguiente. Por tanto, se imponen recortes a lo largo de toda la cadena productiva.

Los recortes suelen ser de orden financiero únicamente. A las

23 O. Lange, 1970.

24 J. Goldmann, 1964

25 Véase, por ejemplo, J. Kornoi, 1979 y P. Dziewulski. 
autoridades centrales de planificación ya no les es posible reconsiderar proyectos en lo individual, salvo aquellos que son de mayor importancia y sólo en términos generales. Los ministerios reciben la orden de recortar la inversión planeada en una suma o un porcentaje globales. Este mensaje se transmite a las instancias inferiores y se interpreta restando fondos a todos los proyectos, más que eliminando algunos. Es así como las reducciones impuestas desde arriba amplían las distorsiones surgidas en las instancias inferiores por subestimar, como ya hemos señalado, los costos de la inversión planeada.

Es claro, pues, que todo plan quinquenal, ya antes de ejecutarse, encierra distorsiones en lo que a inversión se refiere. Con el tiempo, estas fisuras ejercen presión sobre el equilibrio global, o, más concretamente, crean desequilibrio por exceso de demanda en los centros de producción. El desequilibrio aumenta. La subestimación de costos y la sobrestimación de resultados afectan sobre todo al sector de manufactura de bienes de producción y al sector de construcción, porque son éstos los que tienen que hacer frente directamente a la demanda de inversión. Ahora bien, aunque algunas de las nuevas fabricas tomadas en cuenta al hacer el plan puedan no alcanzar la capacidad esperada o ni siquiera hayan comenzado a operar, los gastos que prevé el plan sí se habrán hecho efectivos y los sueldos, premios y bonos se habrán pagado. De esta manera, como haya proyectos de inversión inconclusos en el sector de bienes de consumo y parte de la producción planeada no llegue al mercado, el desequilibrio se transformará en brecha inflacionaria.

El desequilibrio global y la brecha inflacionaria alcanzan un má$x$ imo hacia la mitad del período que abarcan los planes a mediano plazo, es decir, desde principios de los sesenta, los planes quinquenales. Sólo entonces, bajo la presión de un exceso de demanda cada vez mayor, tanto el plan de inversión como el plan de producción son sometidos a revisión minuciosa. Con costos ya dados y con retrasos de entrega imposibles de pasar por alto, se procede, por fin, a recortar proyectos y no únicamente fondos. Muchos de los proyectos son puestos en congelador, aplazando su continuación al próximo período bajo plan a mediano plazo, $y$ otros son suspendidos definitivamente. Es entonces cuando la economía empieza a resentir la carga de costos que no tienen contrapartida en resultados. Todo proyecto congelado o suspendido aparece sólo de un lado de la contabilidad.

Generalmente, durante la segunda mitad del periodo bajo plan no se inicia ningún proyecto de inversión importante. La actividad se concentra en la terminación de proyectos comenzados en fases ante- 
riores. Si dentro de los proyectos finalmente concluidos hay una buena parte que corresponde al sector de bienes de consumo, la brecha inflacionaria deja de aumentar $o$, incluso, decrece hasta cierto punto. Sin embargo, cuando es tan amplia que no puede cerrarse incrementando la oferta, se declaran aumentos de precios para reducir la demanda. Cabe señalar en este punto, aún a sabiendas de que se tomará como prueba meramente empirista, que los aumentos de precios observados suelen estar concentrados en la segunda mitad de los periodos bajo plan a mediano plazo. Al finalizar esta etapa se restablece el equilibrio, o, al menos, el desequilibrio en pequeña escala, y la economía entra en un nuevo ciclo.

He aquí mi "modelo" estandarizado para explicar el ciclo de inversión y la brecha inflacionaria que provoca. La brecha inflacionaria en cuestión es cambiante o fluctuante, no estable, como indica el modelo tradicional.

En efecto, la brecha varía en el tiempo y de un país a otro. Depende, entre otros factores, de la habilidad para recortar proyectos más que fondos al diseñar un plan, de la oportunidad con que se frena la inversión (cuanto más tiempo se tarde, mayor será la presión sobre los precios) y de la rapidez con que se escogen los proyectos a ejecutar o los proyectos a eliminar cuando se reconoce la necesidad de introducir cambios en el plan. Al examinar la magnitud de la brecha inflacionaria no es posible pasar por alto el papel que juegan quienes diseñan la política económica. Sus decisiones, a veces arbitrarias, pueden agudizar el desequilibrio si se traducen en nuevos proyectos ( $y$, por tanto, en mayores gastos) sin cancelar algunos de los aprobados con anterioridad. ${ }^{26}$

\section{AUMENTO EN EL COSTO DE VIDA: INFLACIÓN POR EXCESO DE DEMANDA F INFLACIÓN POR OTRAS CAUSAS}

El carácter expansionista de los sistemas de dirección de la producción nos ha permitido, hasta aquí, establecer una concatenación

26 Polonia, por ejemplo, tiene en su haber dos períodos de intervención arbitraria: el plan 1950-1955 y los años setenta. Los dos planes quinquenales de la década del 70 debieran considerarse como un solo ciclo. Quienes diseñaron entonces la política económica introdujeron demasiados proyectos de poca envergadura $\mathrm{y}$, lo que es más grave, ignoraron señales ominosas. Hacia 1975 no pusieron freno a la inversión ni, por tanto, al desequilibrio. El resultado fue, finalmente, un descenso abrupto y prolongado en la inversión. 
entre plan de inversión a mediano plazo, ciclo de inversión, desequilibrio global y brecha inflacionaria. En esta sección examinaremos ante todo las relaciones múltiples entre brecha inflacionaria e inflación, interpretada ésta como el aumento en el costo de vida.

Hablamos de relaciones múltiples porque, como bien se sabe, en una economía centralmente planificada la brecha inflacionaria no se corivierte espontáneamente en inflación, si por inflación entendemos el aumento del índice de precios al consumidor. Es necesario tener presente que en dicha economía hay un control de precios más o menos generalizado.

En tal contex to, ¿cuáles son las respuestas posibles a un desequilibrio y a una brecha inflacionaria crecientes? Por una parte, evitar que la brecha continúe abriéndose, recortando el programa de inversión y limitando, así, el crecimiento del fondo de salarios en el sector construcción y en el de manufactura de bienes de producción. Por otra parte, cerrar la brecha declarando un aumento único en el nivel de precios. Generalmente, como señalábamos, esta última medida se toma durante la segunda mitad del período bajo plan a mediano plazo, es decir, una vez que la brecha ha llegado a un máximo.

Ni siquiera es seguro que se intente cerrar la brecha mediante aumentos de precios. Desde luego, antes se probarán otros métodos. Por ejemplo, cambiar la composición de los proyectos de inversión finalmente escogidos para terminarse, dando a la producción de bienes de consumo mayor peso del que tuviera al iniciarse el plan.

No es de extrañar, pues, que algunos estudiosos vean en el índice de precios al consumidor, o sea, en el cambio de precios al menudeo, un reflejo muy vago de las presiones inflacionarias subyacentes en una economía centralmente planificada. Janos Kornoi ha señalado, por ejemplo, que la escasez de productos, como manifestación de brecha inflacionaria, es compatible con precios crecientes, estables o decrecientes. ${ }^{27}$

El hecho de que la brecha inflacionaria no se transmita espontáneamente a los precios al consumidor, no significa que no haya otras "correas de transmisión", aunque menós visibles. Para empezar, dicho índice puede subir aunque no aumenten los precios en lo individual. Los estudiosos de la economía húngara ilustran esta posibilidad con tres ejemplos:

27 J. Kornoi, 1979. Véase, también, J. Mujzel, 1974 y J. Sokolowski, 1978. 
- El primero y más claro es el cambio en la composición de productos que forman la canasta básica de bienes de consumo. Al disminuir la oferta de uno de esos bienes, otro más caro puede ganar mercado y generar, con ello, un aumento no registrado en el costo de vida.

- El segundo es el cambio en la calidad de productos. Una baja de calidad, debido al uso de mano de obra peor calificada o de materias primas $y$ bienes intermedios con menores propiedades, es un aumento en el costo de la vida, a menos que los precios registren cambios compensatorios.

- El tercero es la aparición en el mercado de nuevos productos cuyo precio es desproporcionadamente alto frente al de sustitutos cercanos ya disponibles con anterioridad. Cualquier diferencia de precios que sobrepasa una diferencia en calidad o funcionalidad de productos es un aumento de precios. ${ }^{28}$

La expansión que fomenta el sistema de dirección económica, remunerando aumentos de valor y de volumen en la escala productiva, y la "atracción" que ejerce todo producto terminado ${ }^{29}$ abren otro conducto por el que se transmite la brecha inflacionaria. Como hay permanente escasez de repuestos pequeños de bienes de consumo durables, los consumidores se ven obligados a comprar repuestos mayores y más costosos, o incluso nuevos bienes. ${ }^{30}$ Este aumento en el costo de vida es, también, un aumento en los costos de producción, pero los consumidores, a diferencia de las empresas, no tienen la ventaja de operar con un "presupuesto holgado".

Cabe mencionar, por último, la influencia de la llamada "distribución secundaria". El comercio ilegal de productos de oferta muy limitada, bien por debajo del mostrador o bien por canales de distribución no usuales, aumenta el costo de vida a un grado que no es posible cuantificar.

La presencia de estos vínculos múltiples entre brecha inflacionaria y costo de vida me permite concluir que la tesis sobre la falta de transmisión automática entre brecha inflacionaria y precios, aunque válida, carece de importancia para los propósitos de este trabajo. Incluso a falta de cambios espontáneos en precios, hay cambios espontáneos (o fuera de control) en el costo de vida. Por tanto, si el aumento en el costo de vida es inflación, no es ilegítimo relacionar

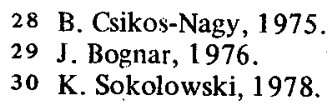


brecha inflacionaria con inflación al analizar las economías centralmente planificadas.

Por lo demás, el exceso de demanda no es el único factor inflacionario, aunque sea, desde luego, el que tiene mayor peso. Esporádicamente, las economías planificadas enfrentan inflación por costos. La correspondencia que deben guardar los salarios de distintas ramas no es compatible con las diferencias en productividad y genera un tipo de inflación por costos que tiene rasgos en común con el proceso ya descrito en este trabajo.

Al ampliar o introducir por primera vez el sistema de salarios por obra terminada, algunas ramas de la industria logran aumentos de productividad considerables y pueden incrementar salarios aceleradamente. Esta medida, sin embargo, afecta los diferenciales de salarios fijados para la economía en conjunto, hasta que los trabajadores de las ramas industriales no favorecidas por el aumento en productividad y de las ramas de servicios demandan mayor remuneración. Los aumentos de salarios finaimente acordados por las autoridades centrales elevan los costos, sobre todo en los servicios. Por ejemplo, el aumento de salarios en el servicio de transporte ferroviario o en los servicios de salud incrementan, pasado cierto tiempo, el precio de pasajes y medicamentos.

Los demás tipos de inflación examinados en este trabajo no parecen afectar a las economías centralmente planificadas, exceptuando aquellos que resultan de situaciones o fenómenos esporádicos (la posguerra $o$, posteriormente, las malas cosechas). Si hay alguna excepción, más que abarcar a todas esas economías, toca a un país en particular, como fue el caso extremo de Polonia durante los setenta y principios de los ochenta.

\section{CONCLUSIONES EN TÉRMINOS COMPARATIVOS Y POLÍTICA ECONÓMICA}

De todas las consideraciones hechas en el trabajo es posible desprender dos conclusiones de carácter más o menos general. Primero, como ya se apuntaba en la introducción, la inflación en economías industrializadas, sean de mercado o planificadas, no tiene una causa única. Los modelos de inflación examinados nos sitúan ante un fenómeno complejo. Segundo, como también se dejaba ver al principio del tra. bajo, las causas de inflación tomadas como más importantes y persistentes son distintas en un tipo de economía que en otro. Por 
curioso que parezca, la inflación de las economías planificadas sigue muy de cerca el modelo tradicional de exceso de demanda, mientras que la inflación en economías de mercado se apega mejor a un modelo fuertemente apoyado en ciertos factores sociales presentes en el mundo occidental industrializado a partir de la posguerra.

Al hablar sobre el peso que tiene el exceso de demanda en una economía y otra no hay que caer en errores. Incluso tratándose del mismo fenómeno, conviene no olvidar que estamos frente a dos sistemas distintos. En la economía de mercado, el exceso de demanda es resultante de procesos bien definidos teóricamente como espontáneos, mientras que en la economía planificada proviene de un tipo de planeación guiada por sistemas de dirección expansionistas. Además, en la economía planificada los desequilibrios cíclicos y la inflación resultante acusan sobreinversión, no subinversión, como en la economía de mercado.

Estas consideraciones son importantes al evaluar la política antiinflacionaria en una economía y otra. Empecemos por la economía de mercado. Aquí, el campo de acción es, en definitiva, muy restringido, tomando en cuenta o no la posibilidad de controlar o manejar la demanda. De hecho, las opciones que más se manejan constituyen los dos extremos de toda la gama de posibilidades.

La primera, favorecida generalmente por quienes creen en la capacidad de autorregulación del mercado, pone de relieve la necesidad de implantar nuevamente la competencia en el sector oligopóli$\operatorname{co}^{31}$ de la economía. La propuesta no va más allá. Es difícil encontrar señalamientos concretos para lograr el objetivo señalado. Por otra parte, si se puede hablar de una tendencia a largo plazo en las economías de mercado, la dirección es hacia el aumento de mercados oligopólicos.

La segunda opción defiende la intervención directa en la empresa, implantando en el conjunto de la economía minuciosos controles de costos y precios del tipo que proponen autores como Galbraith. Una política tan extrema como ésta no tiene antecedentes, más que, si acaso, en tiempos de guerra. Los detalles técnicos del control de precios son bien conocidos. La razón de que esta opción se descarte

31 La corriente del pensamiento (o, para ser más exactos, la corriente filosófica) que cree en la capacidad del mercado para autorregularse olvida que la necesidad de intervenir para favorecer la competencia nace, precisamente, de la falta de autorregulación. El punto en cuestión no es el grado de intervención, sino el tipo de intervención: a saber, directa o indirecta. 
es, precisamente, el grado inaceptable de intervención que entraña. Controles más selectivos han regido, aunque temporalmente, en diversas economías de mercado.

La primera opción parece efectiva, ${ }^{32}$ pero no realista, dada la presencia cada vez mayor de mercados oligopólicos, mientras que la segunda es posible, y, por tanto, realista, pero de eficacia dudosa si pensamos en la inflación provocada por la concatenación de productividad, ganancias, salarios, costos y precios.

En las economías de mercado las presiones inflacionarias a partir de la posguerra provienen de una alta productividad más que de lo contrario. En este contexto, los controles no establecerían ninguna diferencia que justificara intervención. Las demandas de aumento de salarios no irían más lejos que el incremento de productividad, los costos de producción no subirían, etc. La intervención en el proceso de negociación global de salarios no tendría efecto ninguno, puesto que la parte del aumento en productividad que se lograra incorporar al ingreso de los trabajadores no se traduciría en disminución de precios, sino en aumento de ganancias o, vía impuestos, de otro tipo de beneficios generalmente reconocidos. Asi las cosas, la intervención tendría que darse en el proceso de negociación salarial de las ramas industriales con alta productividad.

Examinemos, ahora, el caso de las economías planificadas. Aquí es todavía más difícil encontrar políticas antiinflacionarias formuladas con precisión. Hay que suponer que este vacío nace de la debilidad teórica detrás de la explicación misma del fenómeno. Intentaré plantear una opción que, a mi juicio, haría desaparecer la inflación por exceso de demanda provocada por los ciclos de inversión.

En secciones anteriores hemos hecho hincapié en el carácter endógeno de los ciclos de inversión, asociado al sistema de dirección de las economías planificadas. Es en el sistema, más que en el proceso de inversión, donde hay que implantar mejoras.

Los intentos por restar atractivo absoluto a la inversión nueva no darán resultados del todo satisfactorios, porque, de cualquier manera, la inversión será más atractiva que la innovación como método para acrecentar la producción. Así lo demuestran los resultados

32 Una menor concentración industrial en determinados mercados traerá resultados ciertamente positivos si, al fijar la tasa de aumento de salarios, se tiene en cuenta el peso que representa la estructura oligopólica de los mercados a que corresponde la tasa de crecimiento de productividad más alta. Si, por el contrario, este máximo se toma como un límite natural, la disminución en concentración no será efectiva. 
negativos de varias reformas. El procedimiento correcto es aumentar el atractivo relativo de la innovación frente a la inversión, sobre todo, la inversión nueva. Para ello habría que implantar reformas económicas que desterraran, $o$, al menos, aminoraran el sesgo en contra de la innovación que es característico de los sistemas de dirección vigentes.

El desaliento a la innovación brota de la naturaleza expansionista de esos sistemas. Las reformas que corrijan este carácter frenarán ese sesgo. La segunda etapa de las reformas introducidas en la economía húngara, a partir de 1980, parece encaminada en tal dirección y, por tanto, arrojará evidencia empírica en un futuro no muy distante.

Las consideraciones que hemos hecho sobre la política antiinflacionaria en los dos tipos de economía examinados en el trabajo no nos pueden llevar a ninguna conclusión optimista. En ambos casos, la clase de inflación predominante es, sin duda, atípica, históricamente hablando, $y$, por consiguiente, no encuentra soluciones bien fundamentadas. Tanto en el campo de la teoría como en el de la investigación empírica queda mucho trabajo por hacer si se considera necesario ampliar la gama de soluciones y sustanciarlas mejor.

\section{Bibliografía}

AUJAC, H., "L'inflation, consequence momentaire du comportement des groupes sociaux", Economie appliquee, No. 4, 1950.

AukRuST, O., "PRIMI: A Model of Price and Income Distribution Mechanism of an Open Economy", Review of Income and Wealth, Vol. 14, 1970.

BAJT, A., "Investment Cycles in European Socialist Economies: A Review Article", Journal of Economic Literature, Vol. 9, No. 1, 1971.

BAUER, R., and others, Ekonomia polityczna socjalizmu (Political Economy of Socialism), PWN Publishers, Varsovia, 1972.

BAUMOL, W. J., "Macroeconomics of Unbalanced Growth: The Anatomy of Urban Crisis", American Economic Review, vol. 57, No. 3, 1967.

BEKSIAK, J., "Zjawiska inflacyjne w gospodarce socjalistycznej (Inflationary Phenomena in a Socialist Economy)", Ekonomista, No. 1, 1966 .

BlaIR, J. M., "Inflation in the United States: A Short-Run Target Return Model", en, The Roots of Inflation. ed. por J. M. Blair, B. Franklin and Co., Nueva York, 1975. 
Bognar, J., "A New Foreign Trade Strategy", New Hungarian Quarterly, Vol. 19, No. 70.

Bourva, J., le, "La theorie d'inflation", Economie Applique, No. $5,1950$.

Csi Kos-Nagy, B., "Socialist Price Theory and Price Policy", Akademiai Kiado, Budapest, 1975.

DzIEWUlSKI, P., A. MACIEJEWSKI, "Nierownomiernose inwestowania a ekspansja inwestycyjna (Uneven Investment Pattern and Investment Expansion)", Gospodarka Planowa, No. 4, 1980.

EATWELl, J., J. LlEWELlYN, R. TARLING, "Money Wage Inflation in Industrial Countries", Review of Economic Studies, No. 4, 1974.

Edgren, G., K. O. Faxen, C. E. Odhner, "EFO Model: Wages, Growth and the Distribution of Income", Swedish Journal of Economics, No. 3, septiembre, 1969.

Galbraith, J. K., The Age of Uncertainty, Houghton Mifflin, Nueva York, 1976.

KorNoI, J., Anti-Equilibrium, North-Holland, Amsterdam, 1971

, "Resource-Constrained versus Demand-Constrained Systems", Institute for International Economic Studies, University of Stockholm, Reprint Series, No. 112, 1979.

Kucharski, M. PIEnIAdz, Dochod, proporcje wzrostu (Money, Income, Growth Proportions), PWE Publishers, Varsovia, 1969.

LANGE, O., Theory of Reproduction and Accumulation, PWN Publishers, Varsovia, 1970.

MALISHEv, Y., "Nyekotoryie woprosy tsenoobrazowanya sotsyalististitcheskom khozyaystve (Some Problems of Price Formation in a Socialist Economy)", Voprosy Ekonomiki, No. 3, 1957.

MEANS, G. C., "Simultaneous Inflation and Unemployment: A Challenge to Theory and Policy" (en), The Roots of Inflation... op. cit.

MUjzEL, J., "Zmiany poziomu cen $w$ gospodarce socjalistycznej (Changes in the Level of Prices in a Socialist Economy)", Ekonomista, No. 4, 1974.

Pewzner, J. A., Gosudarstwo w ekonomikie Japonii (State in the Japonese Economy), Nauka Publishers, Moscú, 1976.

- "Rost tsen w japonyi (Growth of Prices in Japan)", Mirowaya ekonomika i myeshdunarodnyie otnoshenyia, No. 4, 1977.

SADZIKOWSKI, W., Ekonomia polityczna kapitalizmu (Political Economy of Capitalism), PWE Publishers, Varsovia, 1977.

SoкоLоwsк1, K., Inflacja (Inflation), Dissertations of the Copernicus University, Torun, 1978.

Strfeten, P., "Wages, Prices and Productivity: Labour and Inflation", Kyklos, 1962, Fasc. 4. 
WILES, P. J. D., The Political Economy of Communism, Cambridge, U.P., Mass, 1962.

WINIECKI, J., "Sources of Inflation in the Western Economies. Some Theoretical Considerations", Maandschrift Economie, No. 11/12, 1978.

-,Mecanisme: Productivite-Profits-Salaires-Cout-Prix- en tant que source de l'inflation dans l'economie de marche", Conference franco-polonaise.

, Inflation et cycle conjoncturel dans les pays capitalistes industrialises, Varsovia, septiembre 1980 (mimeo.).

—_ , "Zwiazki miedzy cyklem inwestycyjnym a inflacja popytowa $w$ gospodarce planowej (Linkages between Investment Cycle and Demand Inflation in a Planned Economy)", Ekonomista, No. 1, 1982. 